\title{
Atténuation du premier mode de torsion d'une structure par un batteur gyroscopique
}

\author{
Philippe Cuvelier $^{1, a}$, Joël Giardi ${ }^{1}$, An Tuan Pham ${ }^{1}$ et Bertrand Auffray ${ }^{2}$ \\ 1 Laboratoire d'Acoustique et Vibration, École Supérieure des Techniques Aéronautiques et de Construction Automobile, \\ 34 rue Victor Hugo, 92300 Levallois-Perret, France \\ 2 PSA Peugeot Citroën, Centre Technique de Vélizy, Route de Gisy, 78943 Vélizy-Villacoublay, France
}

Reçu le 2 octobre 2008, accepté le 28 janvier 2009

\begin{abstract}
Résumé - Cette étude concerne un dispositif antivibratoire actif utilisant un gyroscope pour atténuer les vibrations transmises à une structure mécanique par une source de vibrations. Ce dispositif a un comportement similaire à des batteurs classiques de type masse-ressort, avec certaines spécificités : masse plus faible, gain et plage fréquentielle d'atténuation réglable à masse identique. Le système a été simulé à partir d'un modèle analytique non-linéaire, validé par une maquette à l'échelle 1.
\end{abstract}

Mots clés : Gyroscope / batteur / vibration / torsion / atténuation / mode

\begin{abstract}
Reduction of the first torsional mode of a structure by a gyroscopic damper. In this study an active vibration absorber using a gyroscope to reduce the magnitude of motions of an excited mechanical structure is presented. This device has almost the same behaviour as dynamic vibration absorbers, with some particularities: mass reduced, gain and attenuation frequency range adjustable with the same mass. This system has been simulated using a non linear analytic model, validated by a real scale mock up.
\end{abstract}

Key words: Gyroscope / damper / vibration / torsion / attenuation / mode

\section{Contexte}

Cette communication concerne un dispositif antivibratoire actif utilisant un gyroscope qui atténue les vibrations en torsion d'une structure mécanique.

Le dispositif peut s'appliquer à toute structure mécanique pour traiter des vibrations de torsion et notamment aux véhicules automobiles de type cabriolet.

L'architecture de ces véhicules induit un manque de rigidité en torsion, qui se traduit par une dégradation du confort vibratoire.

La solution classique est d'atténuer les trépidations dues au premier mode de torsion de caisse par l'ajout de systèmes masse-ressort appelés batteurs [1].

C'est le cas par exemple pour les véhicules automobiles de marques commerciales Peugeot 307-CC ou Citroën C3-Pluriel (pour ces deux marques de véhicules, les

\footnotetext{
a Auteur pour correspondance :

pcuvelier@estaca.fr
}

batteurs sont situés sous les longerons, ou longeronnets arrière) ou de marque commerciale Renault Megane-CC (les batteurs sont cachés derrière les feux arrière).

Cette solution, très robuste, a le gros inconvénient d'augmenter la masse du véhicule d'une vingtaine de kg.

Les dispositifs actifs pour atténuer les vibrations comportent habituellement des boucles de contrôle réalisées par des systèmes électroniques qui contribuent à diminuer leur fiabilité. La solution proposée ici, utilise un batteur gyroscopique beaucoup plus léger et non piloté (en boucle ouverte) [2]. Dans le cadre de cette étude, une maquette représentative d'un cabriolet à l'échelle un (même inertie et premier mode propre à $15 \mathrm{~Hz}$ ) a été développée et réalisée dans nos locaux, afin de valider le modèle analytique et de mesurer l'efficacité réelle du dispositif.

Tous les développements théoriques et les simulations ont été recalés sur celle-ci, excitée en sinus balayé par un pot vibrant au travers d'une attaque élastique sur la bande de fréquence de 10 à $20 \mathrm{~Hz}$. 


\section{Nomenclature}

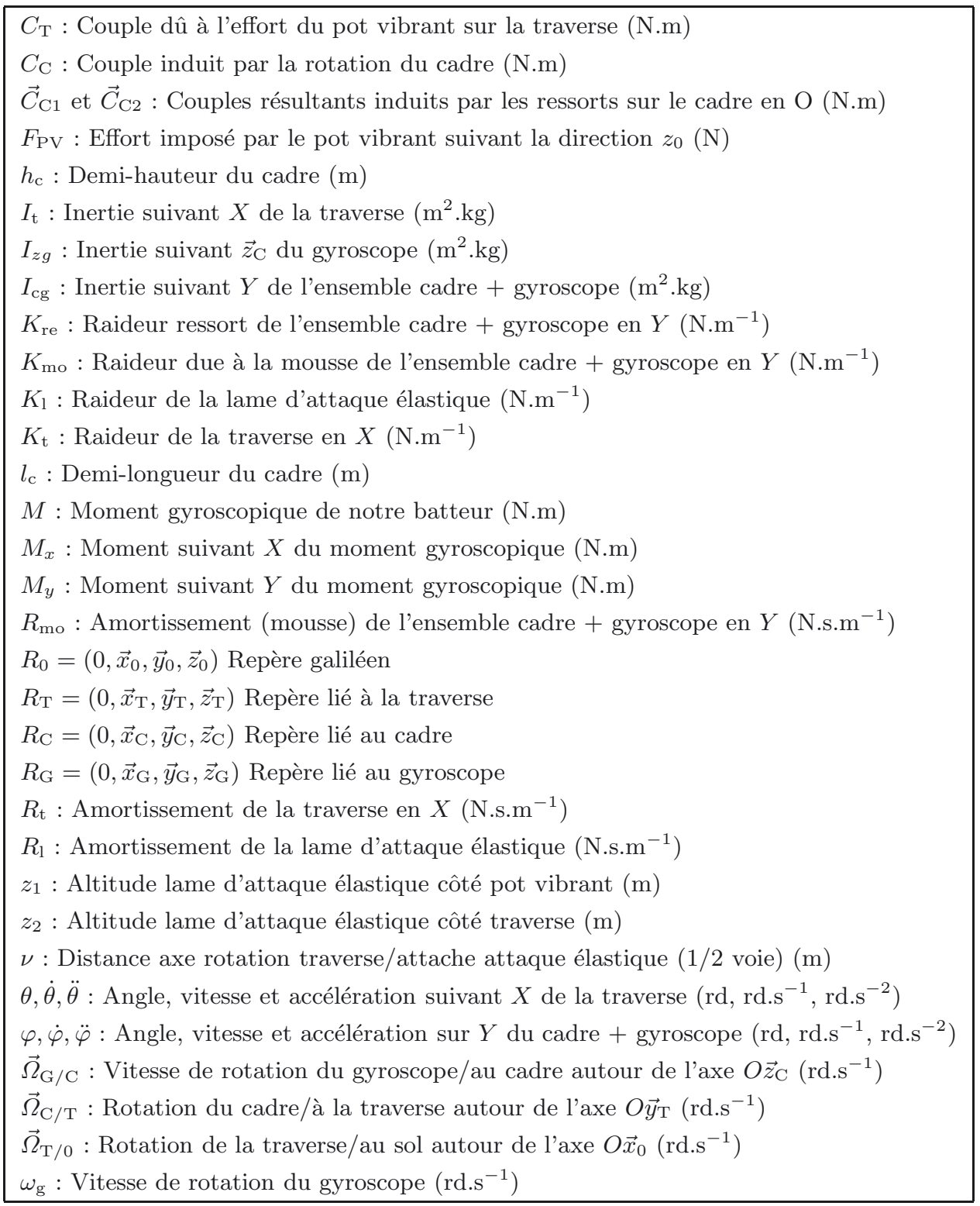

\section{Présentation de la maquette développée pour le cadre de l'étude}

Le gyroscope est placé dans un cadre oscillant amorti, d'axe perpendiculaire à l'axe du mode de torsion et accordé sur la fréquence de ce dernier (Fig. 1).

\subsection{La structure}

Elle est constituée d'une pièce «traverse » fixée au sol par l'intermédiaire de 2 pièces longerons. L'ensemble est dimensionné pour avoir son premier mode de torsion à $15 \mathrm{~Hz}$ suivant l'axe $X$. La pièce « traverse » représente l'inertie de la partie arrière du véhicule, et les pièces « longerons » représentent la raideur du châssis du véhicule (Fig. 2).

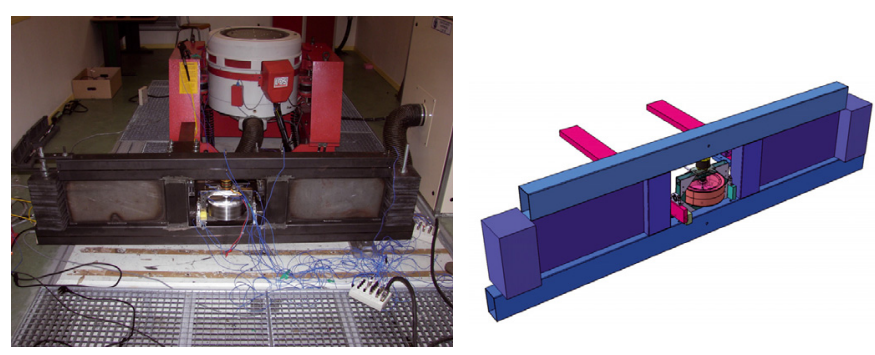

Fig. 1. La maquette.

\subsection{L'actionneur gyroscopique}

Il est constitué d'un « cadre » tournant librement sur des roulements à billes, suivant l'axe $Y$. Dans ce « cadre » est fixé le gyroscope en acier d'une masse de $5 \mathrm{~kg}$ environ, 


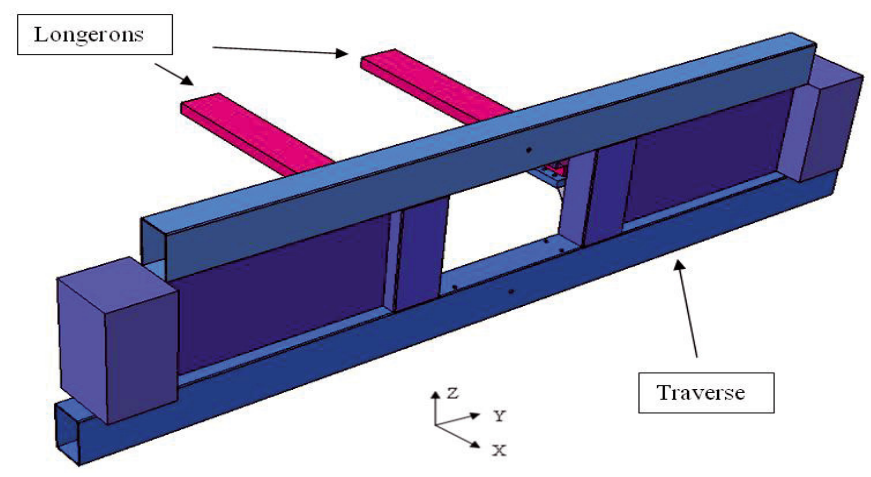

Fig. 2. La structure.

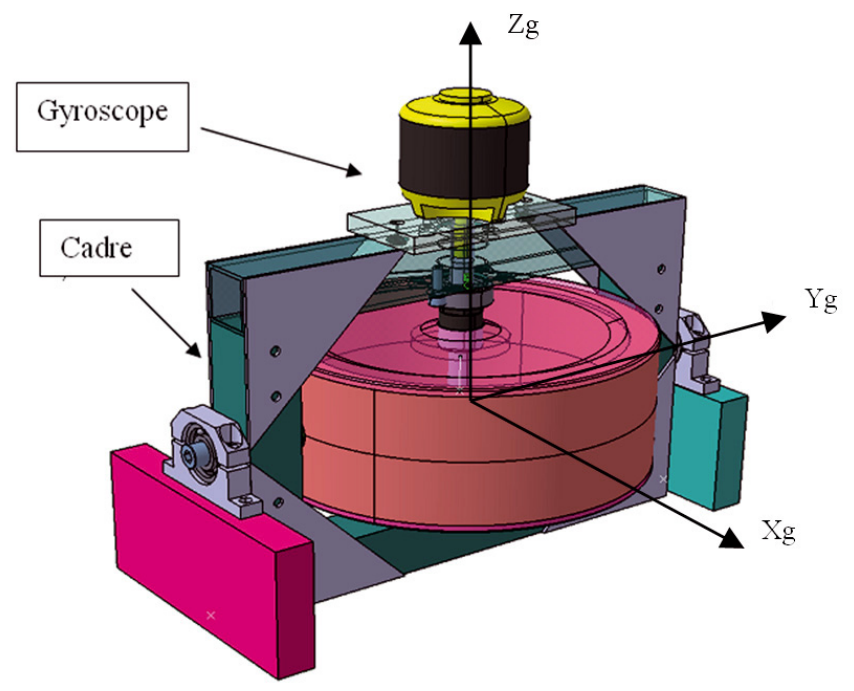

Fig. 3. L'actionneur gyroscopique.

entraîné en rotation par un moteur électrique. Nous avons limité les essais à une vitesse de 9000 tr.min ${ }^{-1}$.

Des ressorts imposent une fréquence propre de $15 \mathrm{~Hz}$ à l'ensemble « cadre + gyroscope », sur l'axe $Y$. L'amortissement du « cadre » est assuré par un dispositif réglable approprié indépendant.

\section{Formalisme théorique}

Le modèle analytique a été développé en considérant les différentes pièces rigides et la vitesse du gyroscope constante.

On étudie le système $\{$ Traverse + Cadre + Gyroscope\} (Fig. 4).

On définit les quatre repères suivants :

- $R_{0}=\left(0, \vec{x}_{0}, \vec{y}_{0}, \vec{z}_{0}\right)$ repère galiléen;

$-R_{\mathrm{T}}=\left(0, \vec{x}_{\mathrm{T}}, \vec{y}_{\mathrm{T}}, \vec{z}_{\mathrm{T}}\right)$ repère lié à la traverse;

$-R_{\mathrm{C}}=\left(0, \vec{x}_{\mathrm{C}}, \vec{y}_{\mathrm{C}}, \vec{z}_{\mathrm{C}}\right)$ repère lié au cadre;

- $R_{\mathrm{G}}=\left(0, \vec{x}_{\mathrm{G}}, \vec{y}_{\mathrm{G}}, \vec{z}_{\mathrm{G}}\right)$ repère lié au gyroscope.

\subsection{Cinématique}

Nous pouvons décomposer notre système sous la forme de 3 rotations :

Rotation du gyroscope par rapport au cadre autour de l'axe $O \vec{z}_{\mathrm{C}}$ (Fig. 5) :

$$
\vec{\Omega}_{\mathrm{G} / \mathrm{C}}=\left(\begin{array}{c}
0 \\
0 \\
\omega_{\mathrm{G}}
\end{array}\right)_{R_{\mathrm{C}}}=\omega_{\mathrm{G}} \vec{z}_{\mathrm{C}}
$$

Rotation du cadre par rapport à la traverse autour de l'axe $O \vec{y}_{\mathrm{T}}$ (Fig. 6) :

$$
\vec{\Omega}_{\mathrm{C} / \mathrm{T}}=\left(\begin{array}{c}
0 \\
\dot{\varphi} \\
0
\end{array}\right)_{R_{\mathrm{T}}}=\dot{\varphi} \vec{y}_{\mathrm{T}}
$$

Rotation de la traverse par rapport au sol autour de l'axe $O \vec{x}_{0}$ (Fig. 7) :

$$
\vec{\Omega}_{\mathrm{T} / 0}=\left(\begin{array}{c}
\dot{\theta} \\
0 \\
0
\end{array}\right)_{R_{0}}=\dot{\theta} \vec{x}_{0}
$$

\subsection{Détermination des moments appliqués sur la traverse en 0}

La traverse est soumise à trois moments :

- $C_{\mathrm{T}}$ le couple dû à l'effort du pot vibrant sur la traverse;

- $C_{\mathrm{C}}$ le couple induit par les ressorts sur le cadre;

- $M$ le couple gyroscopique de notre batteur.

Le couple gyroscopique est considéré comme résistant par rapport à $C_{\mathrm{T}}$ et moteur par rapport à $C_{\mathrm{C}}$. Il se décompose en trois composantes $M_{x}, M_{y}$ et $M_{z}$.

Tous nos moments sont calculés au point $\mathrm{O}$. Après calcul et projection des différents moments (voir annexe A), par application du principe fondamental de la dynamique $\vec{C}_{\mathrm{T}}=I \overrightarrow{\dot{X}}+R \overrightarrow{\dot{X}}+K \vec{X}+\vec{C}_{\mathrm{C}}+\vec{M}$ avec

$$
\vec{X}=\left(\begin{array}{c}
\theta \\
\varphi \\
\psi
\end{array}\right) \quad \text { et } \quad \omega_{\mathrm{G}}=\dot{\psi} .
$$

On obtient dans le repère galiléen : $R_{0}=\left(0, \vec{x}_{0}, \vec{y}_{0}, \vec{z}_{0}\right)$ les équations suivantes :

selon $\vec{x}_{0}$ :

$$
\begin{aligned}
(\nu \cos (\theta)+h \sin (\theta))\left(-R_{1}\left(\dot{z}_{2}-\dot{z}_{1}\right)-K_{1}\left(z_{2}-z_{1}\right)\right) \\
-I_{z \mathrm{~g}} \varpi_{\mathrm{G}} \dot{\varphi} \cos (\varphi)=I t \ddot{\theta}+R t \dot{\theta}+K t \theta
\end{aligned}
$$

selon $\vec{y}_{0}$ :

$$
\begin{aligned}
& I_{z \mathrm{~g}} \varpi_{\mathrm{G}}(\dot{\theta} \cos (\theta) \cos (\varphi)+\dot{\varphi} \sin (\theta) \sin (\varphi)) \\
& \quad-h_{\mathrm{c}}\left[2 h_{\mathrm{c}} \cos (\theta) \cos (\varphi)+l_{\mathrm{c}} \sin (\theta)\right] \\
& \quad \times\left[\left(K_{\mathrm{mo}}+K_{\mathrm{re}}\right) \sin (\varphi)+R_{\mathrm{mo}} \dot{\varphi} \cos (\varphi)\right]=I_{\mathrm{cg}} \ddot{\varphi}
\end{aligned}
$$




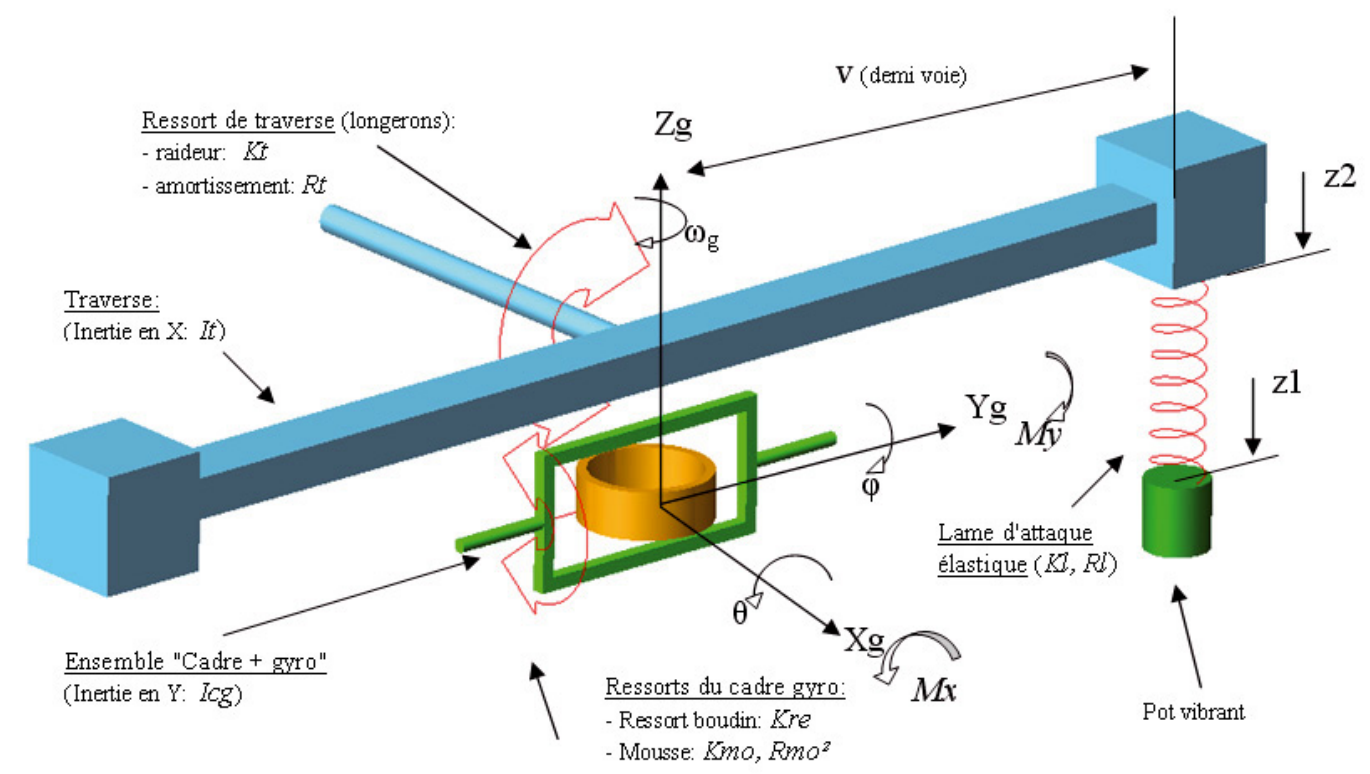

Fig. 4. Modèle traverse/cadre/gyroscope.

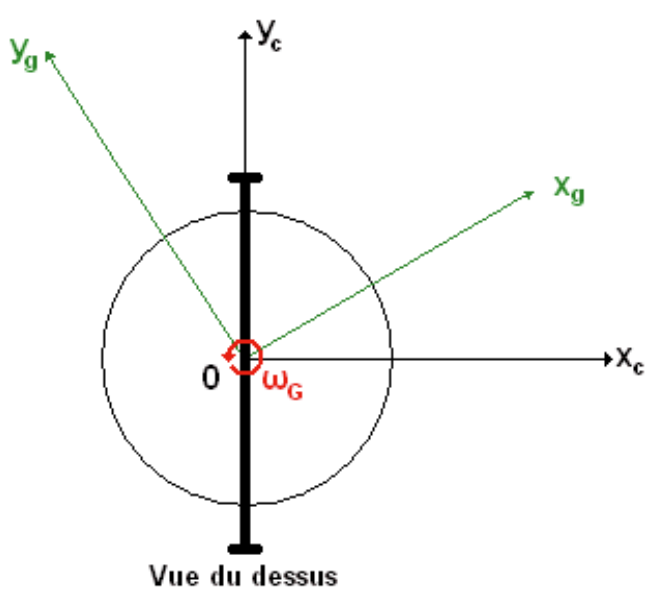

Fig. 5. Rotation du gyro/cadre.

selon $\vec{z}_{0}$ :

$$
\begin{aligned}
& I_{z \mathrm{~g}} \varpi_{\mathrm{G}}(\dot{\theta} \sin (\theta) \cos (\varphi)-\dot{\varphi} \cos (\theta) \sin (\varphi)) \\
& \quad-\left[2 h_{\mathrm{c}} \sin (\theta) \cos (\varphi)+l_{\mathrm{c}} \cos (\theta)\right] \\
& \quad \times\left[\left(K_{\mathrm{mo}}+K_{\mathrm{re}}\right) \sin (\varphi)+R_{\mathrm{mo}} \dot{\varphi} \cos (\varphi)\right]=I_{z \mathrm{~g}} \dot{\omega}_{\mathrm{G}}
\end{aligned}
$$

\section{Linéarisation}

Pour la linéarisation des équations (voir annexe B), nous avons considéré que tous les déplacements étaient «petits» ce qui nous a permis de remplacer tous les cosinus par 1, de remplacer les sinus d'un angle par l'angle

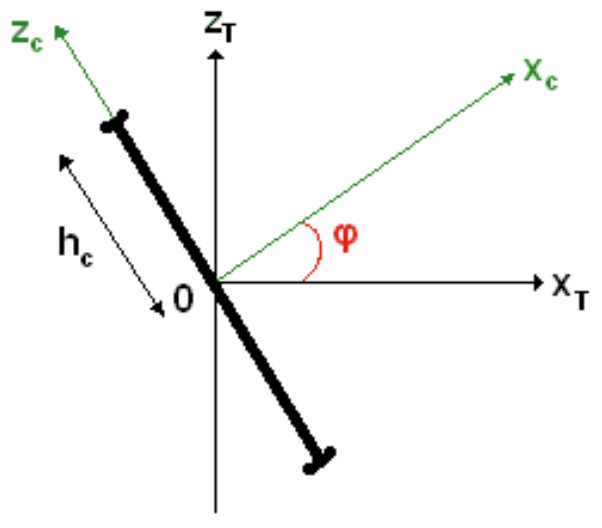

Vue de coté

Fig. 6. Rotation du cadre/traverse.

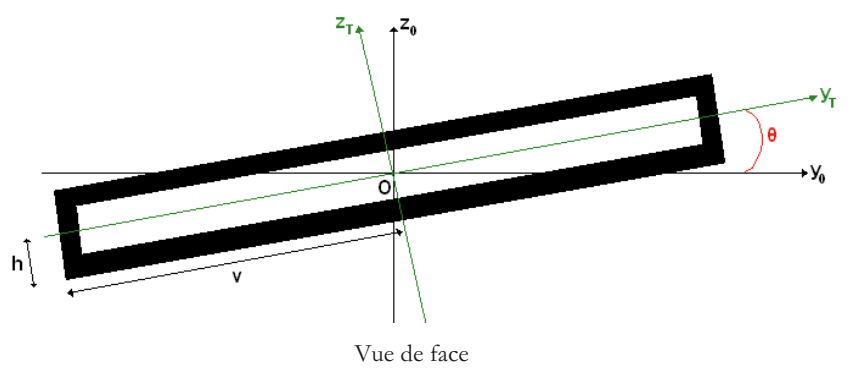

Fig. 7. Rotation de la traverse/sol.

lui-même et de négliger les termes d'ordre supérieur à un par rapport aux termes d'ordre un.

Au final le rapport de la réponse en déplacement $z_{2}$ suivant l'axe $z$ de la traverse sur le déplacement $z_{1}$ de 
l'excitation apportée par le pot vibrant, nous est donné par l'expression :

$$
\begin{aligned}
\frac{z_{2}}{z_{1}}= & \frac{\left(I_{\mathrm{cg}} p^{2}+R_{\mathrm{mo}} p 2 h_{\mathrm{c}}^{2}+\left(K_{\mathrm{mo}}+K_{\mathrm{re}}\right) 2 h_{\mathrm{c}}^{2}\right)}{\left(I t p^{2}+\left(R_{\mathrm{l}} \nu^{2}+R_{\mathrm{t}}\right) p+K_{\mathrm{l}} \nu^{2}+K_{\mathrm{t}}\right)} \\
& \times \frac{\left(R_{\mathrm{l}} \nu p+K_{\mathrm{l}} \nu\right) \nu}{\left(I_{\mathrm{cg}} p^{2}+R_{\mathrm{mo}} p 2 h_{\mathrm{c}}^{2}+\left(K_{\mathrm{mo}}+K_{\mathrm{re}}\right) 2 h_{\mathrm{c}}^{2}\right)+\left(I_{z \mathrm{~g}} \varpi_{\mathrm{G}}\right)^{2} p^{2}}
\end{aligned}
$$

La traverse seule qui n'avait qu'un degré de liberté devient un système à deux degrés de liberté du fait de l'introduction du dispositif cadre+gyroscope (Éq. en $p^{4}$ ).

Ce système présente maintenant deux résonances encadrant une anti-résonance.

La fréquence de l'anti-résonance $\left(\left(I_{\mathrm{cg}} p^{2}+R_{\mathrm{mo}} p 2 h_{\mathrm{c}}^{2}+\right.\right.$ $\left.\left(K_{\mathrm{mo}}+K_{\mathrm{re}}\right) 2 h_{\mathrm{c}}^{2}\right) \approx 0$ ) étant déterminée par la résonance du cadre + gyroscope, il suffit d'accorder celle-ci sur celle de la traverse seule pour réduire fortement l'amplitude du premier mode de torsion de la structure.

Le terme $\left(I_{z \mathrm{~g}} \varpi_{\mathrm{G}}\right)^{2} p^{2}$ va creuser cette dernière quand la vitesse du gyroscope augmentera.

\section{Implémentation dans Simulink du système d'équation différentielle non-linéaire sous forme d'état}

La modélisation sous Simulink permet d'intégrer le système d'équations différentielles non-linéaires et d'obtenir l'évolution du vecteur d'état $X=\left[\begin{array}{c}\dot{\theta} \\ \theta \\ \dot{\varphi} \\ \varphi\end{array}\right]$ en temporel pour tous types de signaux d'excitation harmoniques et non harmoniques.

La transmissibilité $\frac{z_{2}}{z_{1}}$ a été calculée à partir du modèle non-linéaire implémenté dans Simulink, linéarisé sous forme d'état (voir annexe C) autour de zéro pour de petits déplacements.

Cette représentation est équivalente à celle obtenue par la transformée de Laplace (Éq. (4)) et nous avons vérifié par simulation que la réponse dynamique du système linéarisé est identique à celle du système réel pour de petits déplacements.

\section{Comparaison calcul/essais}

\subsection{Protocole d'essais}

La traverse a été excitée en déplacement sinusoïdal d'amplitude crête-crête de $4 \mathrm{~mm}$, de 10 à $20 \mathrm{~Hz}$ au moyen d'un pot vibrant. Les essais ont été réalisés pour différentes vitesses de rotation du gyroscope, différentes valeurs d'amortissement et différentes fréquences propres du cadre. Nous avons mesuré en plusieurs points des accélérations et des forces lors de chaque balayage en fréquence.

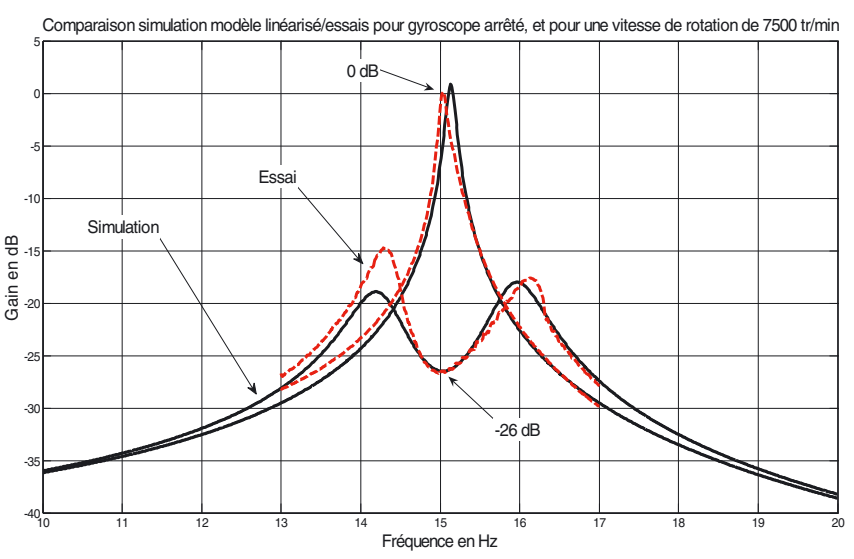

Fig. 8. Comparaison simulation/essai à 0 et 7500 tr.min ${ }^{-1}$.

\subsection{Recalage du modèle analytique}

La traverse seule (c'est-à-dire gyroscope arrêté) a été recalée en fréquence et amortissement. Le comportement du cadre seul a ensuite été identifié en fréquence propre et amortissement, pour différents réglages des amortisseurs et de la vitesse de rotation du gyroscope. Ces deux essais nous ont permis de recaler le modèle analytique traverse + cadre + gyroscope.

Pour différentes vitesses de rotation du gyroscope, nous avons comparé la transmissibilité $\frac{z_{2}}{z_{1}}$ (réponse en déplacement de la traverse sur l'excitation) simulée par le modèle analytique recalé et sa mesure.

Les courbes de la figure 8 vous montrent une comparaison à vitesse nulle et à une vitesse de 7500 tr.min ${ }^{-1}$.

Pour ces essais nous avons calé la fréquence propre du cadre sur celle de la traverse à $15 \mathrm{~Hz}$ et son amortissement à $4 \%$. L'introduction du gyroscope rajoute un degré de liberté qui se traduit par l'apparition d'une antirésonance à $15 \mathrm{~Hz}$, en lieu et place de la résonance de la traverse, encadrée par deux pics comme pour tout batteur classique.

\section{Influence des paramètres}

Nous allons, maintenant, nous intéresser à l'influence des paramètres fondamentaux du dispositif à savoir : la vitesse de rotation du gyroscope, l'amortissement du cadre, et de l'accord en fréquence du mode de la traverse avec celui du cadre, sous-accord, accord et sur-accord.

Les simulations présentées ci-dessous ont été réalisées à partir du modèle linéarisé recalé.

\subsection{Influence de la vitesse de rotation pour un amortissement du cadre de $\xi=4 \%$}

On constate que théoriquement l'atténuation du mode de torsion s'améliore avec la vitesse de rotation du gyroscope, l'anti-résonance s'amplifie avec l'augmentation de la vitesse par le terme $\left(I_{z \mathrm{~g}} \varpi_{\mathrm{G}}\right)^{2} p^{2}$. 


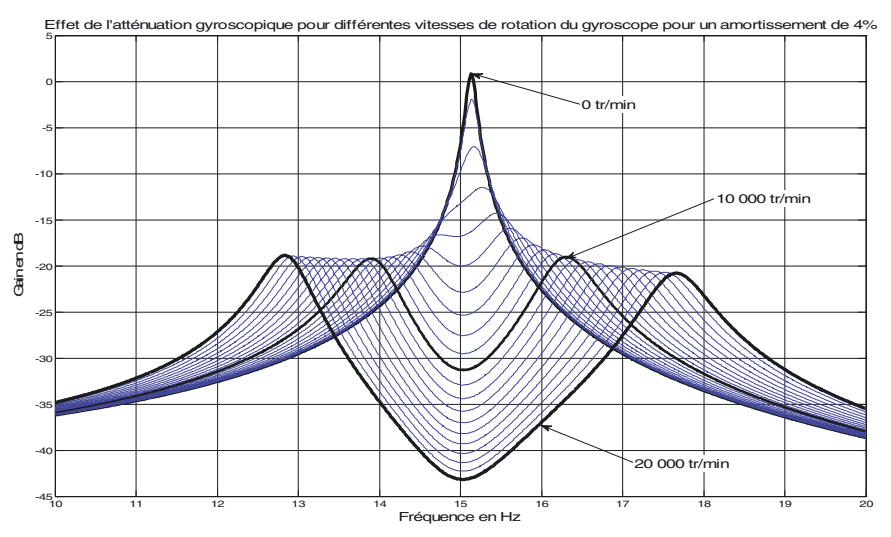

Fig. 9. Influence de la vitesse de rotation.

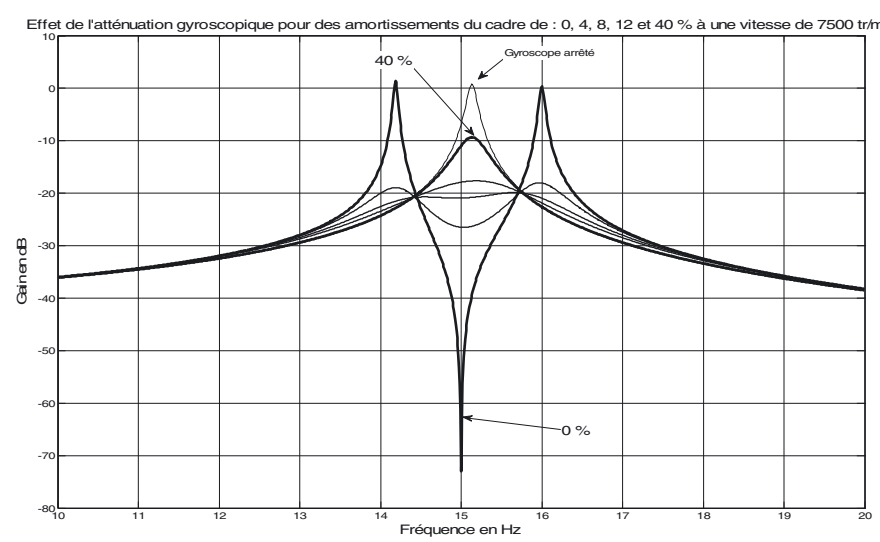

Fig. 10. Influence de l'amortissement.

Les essais confirment cette tendance, nous avons limité la vitesse de rotation jusqu'à 9000 tr. $\mathrm{min}^{-1}$ en raison de différentes contraintes technologiques.

L'anti-résonance se creuse, les résonances s'écartent, leur amplitude reste quasiment constante à partir d'une vitesse de l'ordre de 5000 tr. $\mathrm{min}^{-1}$.

L'amplitude des pics n'évolue pas symétriquement avec l'augmentation de la vitesse de rotation du gyroscope.

\subsection{Influence de l'amortissement du cadre}

L'amortissement du cadre influe considérablement sur le comportement du système.

Le choix optimal de l'amortissement du cadre à une vitesse donnée du gyroscope, sera un compromis entre l'amplitude de l'anti résonance et l'amplitude des pics. Leurs amplitudes augmentent avec la diminution de l'amortissement.

Le terme d'amortissement $R_{\mathrm{mo}} p 2 h_{\mathrm{c}}^{2}$ de l'équation (4) limitant l'amplitude de l'anti-résonance lors de l'accord du mode du cadre avec celui de la traverse, nous observons que pour un amortissement très faible, elle devient très grande et que pour un amortissement de l'ordre de $40 \%$ l'anti-résonance disparaît (Fig. 10).

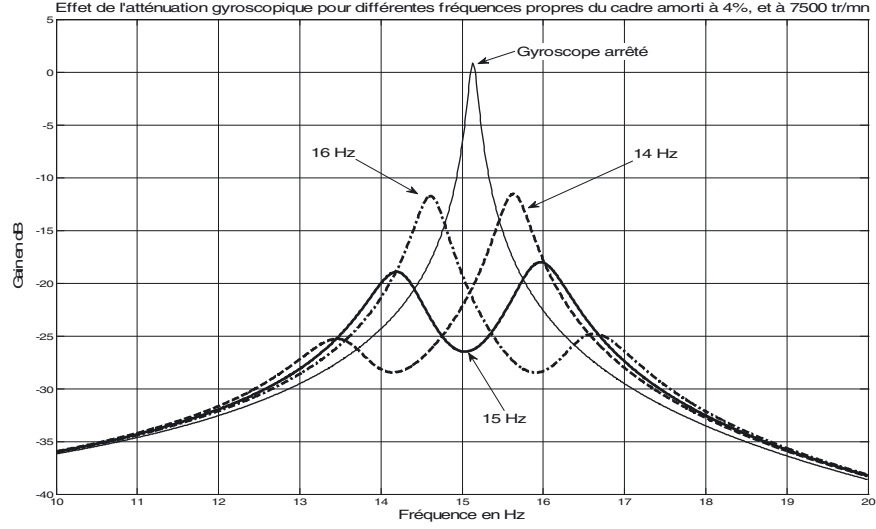

Fig. 11. Influence de la fréquence propre du cadre.

\subsection{Influence de la fréquence propre du cadre}

L'atténuation gyroscopique est maximale quand la fréquence propre du cadre est accordée à la fréquence propre de la structure à corriger (ici la traverse à $15 \mathrm{~Hz}$ ) comme l'a montré l'équation (4).

En cas de désaccord, les pics deviennent dissymétriques et l'atténuation du mode de la traverse est dégradée. Les essais ont montré que sur notre maquette, la fréquence propre du cadre dépend de la vitesse de rotation du gyroscope, ce qui peut désaccorder le système (Fig. 11).

\section{Conclusions}

Les résultats théoriques confortés par les mesures, nous font apparaître que la compensation du premier mode de torsion de la traverse peut être excellente avec un atténuateur gyroscopique plus léger qu'un batteur classique.

Sous l'effet de la compensation gyroscopique la résonance du premier mode de torsion de la traverse se retrouve atténuée (anti-résonance) et encadrée par deux modes comme cela se produit avec des batteurs classiques.

Nous avons obtenu, sur notre maquette à l'échelle un, une atténuation de $26 \mathrm{~dB}$.

L'amortissement rajouté au niveau du ressort de l'ensemble cadre + gyroscope est d'une importance fondamentale en ce qui concerne l'efficacité et la robustesse du système de compensation de mode.

L'importance et l'allure des pics encadrant l'anti résonance peuvent être continûment modifiées par le biais de l'amortissement du cadre, sans modifier la structure ni la masse de l'ensemble.

Le choix de l'amortissement du cadre dépendra du type d'application. Un gain de masse important pourrait être obtenu avec des vitesses de rotation plus élevées.

Les caractéristiques dynamiques de ce dispositif sont facilement modifiables sur une grande plage de réglages sans modification mécanique. 


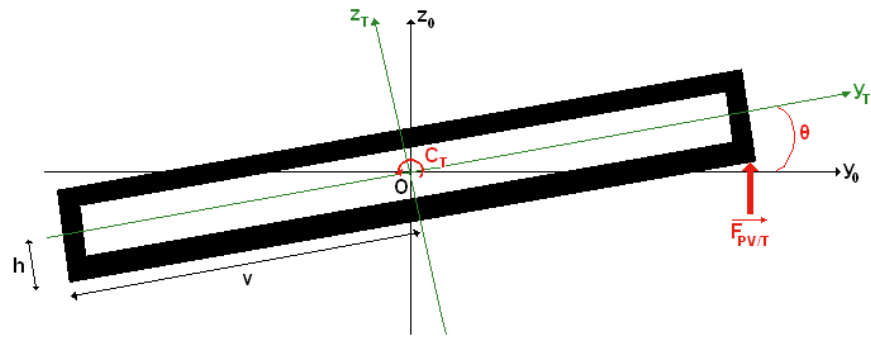

Fig. A1. Moment $C_{\mathrm{T}}$ exercé par le pot sur la traverse.

Ce dispositif qui présente de nombreuses et intéressantes spécificités par rapport aux batteurs classique reste, malgré tout, relativement complexe dans sa mise en ouvre.

Remerciements. Toute l'équipe remercie pour son soutien financier PSA ainsi que Michel Mailly, Responsable des ateliers de l'ESTACA, pour sa collaboration à la réalisation de la maquette.

\section{Annexe $A$ : Mise en équation du batteur-gyroscopique}

\section{Détermination du moment $C_{\mathrm{T}}$ (Fig. A1)}

Par définition, $\vec{C}_{\mathrm{T}}=\vec{F}_{\mathrm{PV} / \mathrm{T}} \wedge\left(h \vec{z}_{\mathrm{T}}-\nu \vec{y}_{\mathrm{T}}\right)[3,4]$ avec $\vec{y}_{\mathrm{T}}=\cos (\theta) \vec{y}_{0}+\sin (\theta) \vec{z}_{0}$ et $\vec{z}_{\mathrm{T}}=\cos (\theta) \vec{z}_{0}-\sin (\theta) \vec{y}_{0}$.

On pose : $F_{\mathrm{PV}}=$ Effort imposé par le pot vibrant suivant la direction $z_{0}$ (Fig. A2).

$\theta$ étant faible, on suppose la composante $\vec{F}_{\mathrm{PV} / \mathrm{T}} \vec{y}_{0}$ négligeable devant $\vec{F}_{\mathrm{PV} / \mathrm{T}} \vec{z}_{0}$. En projetant sur $z_{0}$, on a donc : $\vec{F}_{\mathrm{PV} / \mathrm{T}}=-R_{\mathrm{l}}\left(\dot{z}_{2}-\dot{z}_{1}\right) \vec{z}_{0}-K_{\mathrm{l}}\left(z_{2}-z_{1}\right) \vec{z}_{0}$

$$
\begin{aligned}
& \vec{C}_{\mathrm{T}}=\left(\begin{array}{c}
0 \\
0 \\
-R_{1}\left(\dot{z}_{2}-\dot{z}_{1}\right)-K_{\mathrm{l}}\left(z_{2}-z_{1}\right)
\end{array}\right) \\
& \wedge\left(\begin{array}{c}
0 \\
-\nu \cos (\theta)-h \sin (\theta) \\
-\nu \sin (\theta)+h \cos (\theta)
\end{array}\right) \\
& \vec{C}_{\mathrm{T}}=(v \cos (\theta)+h \sin (\theta))\left(-R_{\mathrm{l}}\left(\dot{z}_{2}-\dot{z}_{1}\right)-K_{1}\left(z_{2}-z_{1}\right)\right) \vec{x}_{0}
\end{aligned}
$$

\section{Détermination du couple gyroscopique $M$ (Fig. A3)}

Par définition, le couple gyroscopique s'exprime par la relation : $\vec{M}=I_{z \mathrm{~g}} \vec{\Omega}_{\mathrm{G} / \mathrm{C}} \wedge \vec{\Omega}_{\mathrm{C} / 0}[5]$, avec $I_{z \mathrm{~g}}$ l'inertie

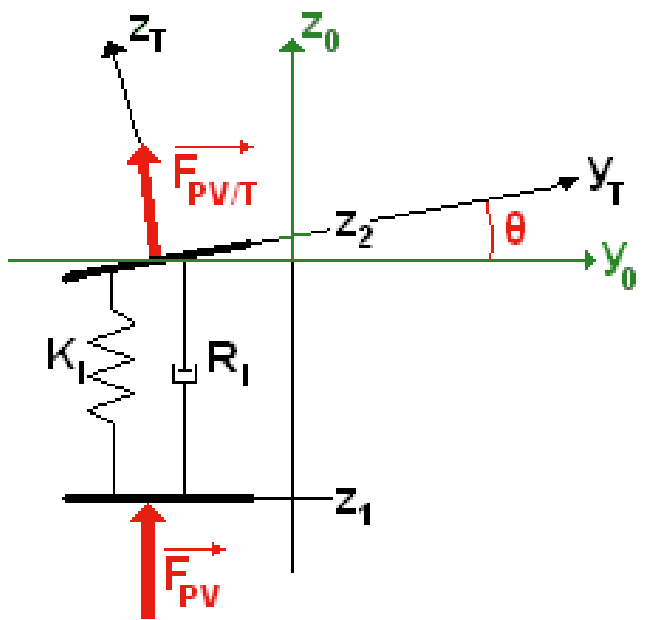

Fig. A2. Détail de l'attaque élastique.

suivant $\vec{z}_{\mathrm{C}}$ du gyroscope. $I_{z \mathrm{~g}}, \vec{\Omega}_{\mathrm{G} / \mathrm{C}}$ et $\vec{\Omega}_{\mathrm{C} / 0}$ sont exprimés dans le repère $R_{0}$

$$
\begin{aligned}
\vec{\Omega}_{\mathrm{C} / 0} & =\dot{\varphi} \vec{y}_{\mathrm{T}}+\dot{\theta} \vec{x}_{0}=\left(\begin{array}{c}
\dot{\theta} \\
\dot{\varphi} \cos (\theta) \\
\dot{\varphi} \sin (\theta)
\end{array}\right)_{R_{0}} \\
\vec{\Omega}_{\mathrm{G} / \mathrm{C}} & =\omega_{\mathrm{G}} \vec{z}_{\mathrm{C}} \\
\text { Or, } \vec{z}_{\mathrm{C}} & =\cos (\varphi) \vec{z}_{\mathrm{T}}-\sin (\varphi) \vec{x}_{\mathrm{T}} \\
\vec{z}_{\mathrm{C}} & =-\sin (\varphi) \vec{x}_{0}-\sin (\theta) \cos (\varphi) \vec{y}_{0}+\cos (\theta) \cos (\varphi) \vec{z}_{0} .
\end{aligned}
$$

Donc, on a :

$$
\vec{\Omega}_{\mathrm{G} / \mathrm{C}}=\varpi_{\mathrm{G}}\left(\begin{array}{c}
-\sin (\varphi) \\
-\sin (\theta) \cos (\varphi) \\
\cos (\theta) \cos (\varphi)
\end{array}\right)_{R_{0}}
$$

et

$$
\vec{M}=I_{z \mathrm{~g}} \varpi_{\mathrm{G}}\left(\begin{array}{c}
-\sin (\varphi) \\
-\sin (\theta) \cos (\varphi) \\
\cos (\theta) \cos (\varphi)
\end{array}\right)_{R_{0}} \wedge\left(\begin{array}{c}
\dot{\theta} \\
\dot{\varphi} \cos (\theta) \\
\dot{\varphi} \sin (\theta)
\end{array}\right)_{R_{0}}
$$

Le couple gyroscopique s'exprime donc ainsi :

$$
\begin{aligned}
\vec{M} & =\left(\begin{array}{c}
M_{x} \\
M_{y} \\
M_{z}
\end{array}\right) \\
& =I_{z \mathrm{~g}} \varpi_{\mathrm{G}}\left(\begin{array}{c}
-\dot{\varphi} \cos (\varphi)\left(\sin ^{2}(\theta)+\cos ^{2}(\theta)\right) \\
\dot{\theta} \cos (\theta) \cos (\varphi)+\dot{\varphi} \sin (\theta) \sin (\varphi) \\
\dot{\theta} \sin (\theta) \cos (\varphi)-\dot{\varphi} \cos (\theta) \sin (\varphi)
\end{array}\right)_{R_{0}}-\dot{\varphi} \cos (\varphi) \\
& =I_{z \mathrm{~g}} \varpi_{\mathrm{G}}\left(\begin{array}{c}
\dot{\theta} \cos (\theta) \cos (\varphi)+\dot{\varphi} \sin (\theta) \sin (\varphi) \\
\dot{\theta} \sin (\theta) \cos (\varphi)-\dot{\varphi} \cos (\theta) \sin (\varphi)
\end{array}\right)
\end{aligned}
$$




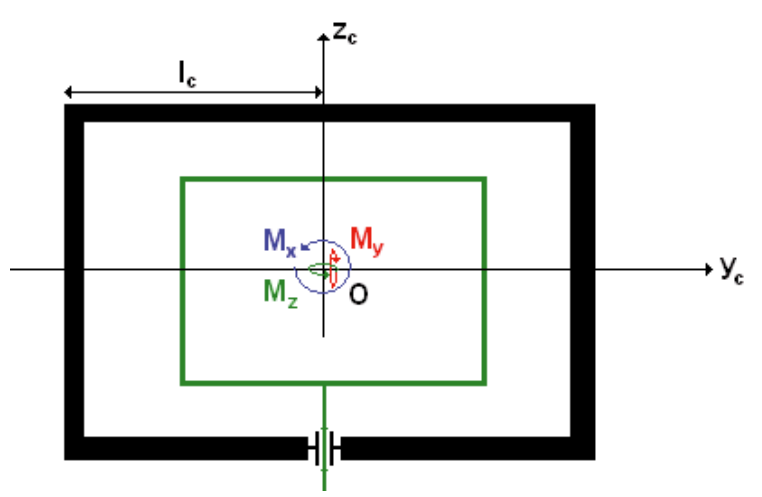

Fig. A3.

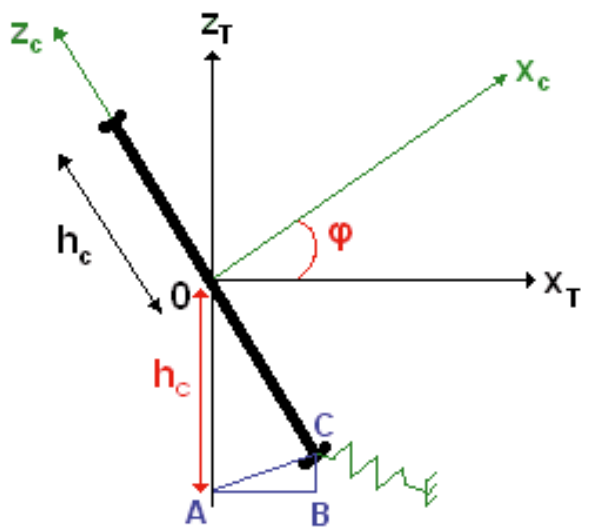

Fig. A4.

Dans l'hypothèse de petits angles, on pose $\cos (X)=1$ et $\sin (X)=0$. On obtient :

$$
\vec{M}=I_{z \mathrm{~g}} \varpi_{\mathrm{G}}\left(\begin{array}{c}
-\dot{\varphi} \\
\dot{\theta} \\
0
\end{array}\right)_{R_{0}}
$$

\section{Détermination du moment $C_{C}$ induit par les ressorts sur le cadre (Fig. A4).}

On appelle $l_{\mathrm{c}}$ la demi-longueur du cadre et $h_{\mathrm{c}}$ la demihauteur du cadre.

La distance $A B$ est l'allongement du ressort 1 selon $\vec{x}_{\mathrm{t}}$ tel que $A B=h_{\mathrm{c}} \sin (\varphi)$ La distance $B C$ est l'allongement du ressort 1 selon $\vec{z}_{\mathrm{t}}$ tel que $B C=h_{\mathrm{c}}(1-\cos (\varphi))$

On peut ainsi exprimer l'effort $\vec{F}_{1}$ exercé par le ressort 1 sur le cadre.

$$
\begin{aligned}
& \vec{F}_{1}=\left(\begin{array}{c}
-\left(K_{\mathrm{mo}}+K_{\mathrm{re}}\right)\left(h_{\mathrm{c}} \sin (\varphi)\right)-R_{\mathrm{mo}}\left(h_{\mathrm{c}} \sin (\varphi)\right)^{\prime} \\
0 \\
-h_{\mathrm{c}}\left(K_{\mathrm{mo}}+K_{\mathrm{re}}\right)(1-\cos (\varphi))-h_{\mathrm{c}} R_{\mathrm{mo}}(1-\cos (\varphi))^{\prime}
\end{array}\right)_{R_{\mathrm{T}}} \\
& \vec{F}_{1}=h_{\mathrm{c}}\left(\begin{array}{c}
-\left(K_{\mathrm{mo}}+K_{\mathrm{re}}\right) \sin (\varphi)-R_{\mathrm{mo}} \dot{\varphi} \cos (\varphi) \\
0 \\
-\left(K_{\mathrm{mo}}+K_{\mathrm{re}}\right)(1-\cos (\varphi))-R_{\mathrm{mo}} \dot{\varphi} \sin (\varphi)
\end{array}\right)_{R_{\mathrm{T}}}
\end{aligned}
$$

Par le même raisonnement, nous exprimons l'effort $\vec{F}_{2}$ exercé par le ressort 2 (non représenté sur le schéma) sur le cadre.

$$
\begin{aligned}
& \vec{F}_{2}=\left(\begin{array}{c}
-\left(K_{\mathrm{mo}}+K_{\mathrm{re}}\right)\left(h_{\mathrm{c}} \sin (\varphi)\right)-R_{\mathrm{mo}}\left(h_{\mathrm{c}} \sin (\varphi)\right)^{\prime} \\
0 \\
h_{\mathrm{c}}\left(K_{\mathrm{mo}}+K_{\mathrm{re}}\right)(1-\cos (\varphi))+h_{\mathrm{c}} R_{\mathrm{mo}}(1-\cos (\varphi))^{\prime}
\end{array}\right)_{R_{\mathrm{T}}} \\
& \vec{F}_{2}=h_{\mathrm{c}}\left(\begin{array}{c}
-\left(K_{\mathrm{mo}}+K_{\mathrm{re}}\right) \sin (\varphi)-R_{\mathrm{mo}} \dot{\varphi} \cos (\varphi) \\
0 \\
\left(K_{\mathrm{mo}}+K_{\mathrm{re}}\right)(1-\cos (\varphi))+R_{\mathrm{mo}} \dot{\varphi} \sin (\varphi)
\end{array}\right)_{R_{\mathrm{T}}}
\end{aligned}
$$

Nous faisons l'hypothèse que l'épaisseur du cadre est négligeable devant les autres dimensions. On pose donc que la composante selon $\vec{x}_{\mathrm{c}}$ de la distance OC est nulle.

Calculons les couples résultants $\vec{C}_{\mathrm{C} 1}$ et $\vec{C}_{\mathrm{C} 2}$ induits par les ressorts sur le cadre en $\mathrm{O}$.

Pour le ressort 1 , on a :

$$
\begin{aligned}
\vec{C}_{\mathrm{C} 1} & =\vec{F}_{1} \wedge\left(h_{\mathrm{c}} \vec{z}_{\mathrm{c}}+l_{\mathrm{c}} \vec{y}_{\mathrm{c}}\right) \\
\vec{y}_{\mathrm{c}} & =\vec{y}_{\mathrm{T}} \text { et } \vec{z}_{\mathrm{C}}=\cos (\varphi) \vec{z}_{\mathrm{T}}-\sin (\varphi) \vec{x}_{\mathrm{T}} \\
\vec{C}_{\mathrm{C} 1} & =h_{\mathrm{c}}\left(\begin{array}{c}
-\left(K_{\mathrm{mo}}+K_{\mathrm{re}}\right) \sin (\varphi)-R_{\mathrm{mo}} \dot{\varphi} \cos (\varphi) \\
0 \\
-\left(K_{\mathrm{mo}}+K_{\mathrm{re}}\right)(1-\cos (\varphi))-R_{\mathrm{mo}} \dot{\varphi} \sin (\varphi)
\end{array}\right)_{R_{\mathrm{T}}}
\end{aligned}
$$

$$
\wedge\left(\begin{array}{c}
-h_{\mathrm{c}} \sin (\varphi) \\
l_{\mathrm{c}} \\
h_{\mathrm{c}} \cos (\varphi)
\end{array}\right)_{R_{\mathrm{T}}}
$$

$\vec{C}_{\mathrm{C} 1}=$

$$
h_{\mathrm{c}}\left(\begin{array}{c}
l_{\mathrm{c}}\left[\left(K_{\mathrm{mo}}+K_{\mathrm{re}}\right)(1-\cos (\varphi))+R_{\mathrm{mo}} \dot{\varphi} \sin (\varphi)\right] \\
h_{\mathrm{c}} \sin (\varphi)\left[\left(K_{\mathrm{mo}}+K_{\mathrm{re}}\right)(1-\cos (\varphi))+R_{\mathrm{mo}} \dot{\varphi} \sin (\varphi)\right] \\
+h_{\mathrm{c}} \cos (\varphi)\left[\left(K_{\mathrm{mo}}+K_{\mathrm{re}}\right) \sin (\varphi)+R_{\mathrm{mo}} \dot{\varphi} \cos (\varphi)\right] \\
-l_{\mathrm{c}}\left[\left(K_{\mathrm{mo}}+K_{\mathrm{re}}\right) \sin (\varphi)+R_{\mathrm{mo}} \dot{\varphi} \cos (\varphi)\right]
\end{array}\right)_{R_{\mathrm{T}}}
$$

De même, le moment du ressort opposé s'exprime comme suit :

$$
\begin{aligned}
\vec{C}_{\mathrm{C} 2}= & \vec{F}_{2} \wedge\left(h_{\mathrm{c}} \vec{z}_{\mathrm{c}}+l_{\mathrm{c}} \vec{y}_{\mathrm{c}}\right) \\
\vec{C}_{\mathrm{C} 2}= & h_{\mathrm{c}}\left(\begin{array}{c}
-\left(K_{\mathrm{mo}}+K_{\mathrm{re}}\right) \sin (\varphi)-R_{\mathrm{mo}} \dot{\varphi} \cos (\varphi) \\
0 \\
\left(K_{\mathrm{mo}}+K_{\mathrm{re}}\right)(1-\cos (\varphi))+R_{\mathrm{mo}} \dot{\varphi} \sin (\varphi)
\end{array}\right)_{R_{\mathrm{T}}} \\
& \wedge\left(\begin{array}{c}
-h_{\mathrm{c}} \sin (\varphi) \\
l_{\mathrm{c}} \\
h_{\mathrm{c}} \cos (\varphi)
\end{array}\right)_{R_{\mathrm{T}}}
\end{aligned}
$$




$$
\frac{\theta}{z_{1}}=\frac{\left(I_{\mathrm{cg}} p^{2}+R_{\mathrm{mo}} p 2 h_{\mathrm{c}}^{2}+\left(K_{\mathrm{mo}}+K_{\mathrm{re}}\right) 2 h_{\mathrm{c}}^{2}\right)\left(R_{\mathrm{l}} \nu p+K_{1} \nu\right)}{\left(I t p^{2}+\left(R_{\mathrm{l}} \nu^{2}+R_{\mathrm{t}}\right) p+K_{1} \nu^{2}+K_{\mathrm{t}}\right)\left(I_{\mathrm{cg}} p^{2}+R_{\mathrm{mo}} p 2 h_{\mathrm{c}}^{2}+\left(K_{\mathrm{mo}}+K_{\mathrm{re}}\right) 2 h_{\mathrm{c}}^{2}\right)+\left(I_{z \mathrm{~g}} \varpi_{\mathrm{G}}\right)^{2} p^{2}}
$$

$\vec{C}_{\mathrm{C} 2}=$

$$
h_{\mathrm{c}}\left(\begin{array}{c}
-l_{\mathrm{c}}\left[\left(K_{\mathrm{mo}}+K_{\mathrm{re}}\right)(1-\cos (\varphi))+R_{\mathrm{mo}} \dot{\varphi} \sin (\varphi)\right] \\
-h_{\mathrm{c}} \sin (\varphi)\left[\left(K_{\mathrm{mo}}+K_{\mathrm{re}}\right)(1-\cos (\varphi))+R_{\mathrm{mo}} \dot{\varphi} \sin (\varphi)\right] \\
+h_{\mathrm{c}} \cos (\varphi)\left[\left(K_{\mathrm{mo}}+K_{\mathrm{re}}\right) \sin (\varphi)+R_{\mathrm{mo}} \dot{\varphi} \cos (\varphi)\right] \\
-l_{\mathrm{c}}\left[\left(K_{\mathrm{mo}}+K_{\mathrm{re}}\right) \sin (\varphi)+R_{\mathrm{mo}} \dot{\varphi} \cos (\varphi)\right]
\end{array}\right)_{R_{\mathrm{T}}}
$$

On note $\vec{C}_{\mathrm{c}}=\vec{C}_{\mathrm{C} 1}+\vec{C}_{\mathrm{C} 2}$

$\vec{C}_{\mathrm{C}}=$

$$
h_{\mathrm{c}}\left(\begin{array}{c}
0 \\
2 h_{\mathrm{c}} \cos (\varphi)\left[\left(K_{\mathrm{mo}}+K_{\mathrm{re}}\right) \sin (\varphi)+R_{\mathrm{mo}} \dot{\varphi} \cos (\varphi)\right] \\
-l_{\mathrm{c}}\left[\left(K_{\mathrm{mo}}+K_{\mathrm{re}}\right) \sin (\varphi)+R_{\mathrm{mo}} \dot{\varphi} \cos (\varphi)\right]
\end{array}\right)_{R_{\mathrm{T}}}
$$

On repasse dans le repère galiléen avec les relations

$$
\begin{aligned}
& \vec{x}_{\mathrm{T}}=x_{0} \\
& \vec{y}_{\mathrm{T}}=\cos (\theta) \vec{y}_{0}+\sin (\theta) \vec{z}_{0} \\
& \vec{z}_{\mathrm{T}}=\cos (\theta) \vec{z}_{0}-\sin (\theta) \vec{y}_{0}
\end{aligned}
$$

On obtient ainsi :

$$
\vec{C}_{\mathrm{C}}=h_{\mathrm{c}}\left(\begin{array}{c}
0 \\
{\left[2 h_{\mathrm{c}} \cos (\theta) \cos (\varphi)+l_{\mathrm{c}} \sin (\theta)\right]} \\
\times\left[\left(K_{\mathrm{mo}}+K_{\mathrm{re}}\right) \sin (\varphi)+R_{\mathrm{mo}} \dot{\varphi} \cos (\varphi)\right] \\
{\left[-2 h_{\mathrm{c}} \sin (\theta) \cos (\varphi)-l_{\mathrm{c}} \cos (\theta)\right]} \\
\times\left[\left(K_{\mathrm{mo}}+K_{\mathrm{re}}\right) \sin (\varphi)+R_{\mathrm{mo}} \dot{\varphi} \cos (\varphi)\right]
\end{array}\right)_{R_{0}}
$$

\section{Annexe B : Linéarisation des équations}

Nous obtenons suivant les différents axes, les expressions simplifiées suivantes :

selon $\vec{x}_{0}$ :

$$
\begin{gathered}
(\nu \cos (\theta)+h \sin (\theta))\left(-R_{\mathrm{l}}\left(\dot{z}_{2}-\dot{z}_{1}\right)-K_{\mathrm{l}}\left(z_{2}-z_{1}\right)\right) \\
-I_{z \mathrm{~g}} \varpi_{\mathrm{G}} \dot{\varphi} \cos (\varphi)=I t \ddot{\theta}+R t \dot{\theta}+K t \theta \\
(\nu+h \theta)\left(-R_{\mathrm{l}}\left(\dot{z}_{2}-\dot{z}_{1}\right)-K_{\mathrm{l}}\left(z_{2}-z_{1}\right)\right)-I_{z \mathrm{~g}} \varpi_{\mathrm{G}} \dot{\varphi}= \\
I t \ddot{\theta}+R t \dot{\theta}+K t \theta \\
I t \ddot{\theta}=(\nu+h \theta)\left(-R_{\mathrm{l}}\left(\nu \dot{\theta}-\dot{z}_{1}\right)-K_{\mathrm{l}}\left(\nu \theta-z_{1}\right)\right) \\
-I_{z \mathrm{~g}} \varpi_{\mathrm{G}} \dot{\varphi}-R t \dot{\theta}-K t \theta
\end{gathered}
$$

$I t \ddot{\theta}=-R_{\mathrm{l}}\left(\nu \dot{\theta}-\dot{z}_{1}\right) \nu-K_{1}\left(\nu \theta-z_{1}\right) \nu-I_{z \mathrm{~g}} \varpi_{\mathrm{G}} \dot{\varphi}-R t \dot{\theta}-K t \theta$

$I t \ddot{\theta}=-R_{1}\left(\nu \dot{\theta}-\dot{z}_{1}\right) \nu-K_{1}\left(\nu \theta-z_{1}\right) \nu-I_{z \mathrm{~g}} \varpi_{\mathrm{G}} \dot{\varphi}-R t \dot{\theta}-K t \theta$

selon $\vec{y}_{0}$ :

$$
\begin{aligned}
& I_{z \mathrm{~g}} \varpi_{\mathrm{G}}(\dot{\theta} \cos (\theta) \cos (\varphi)+\dot{\varphi} \sin (\theta) \sin (\varphi)) \\
& \quad-h_{\mathrm{c}}\left[2 h_{\mathrm{c}} \cos (\theta) \cos (\varphi)+l_{\mathrm{c}} \sin (\theta)\right] \\
& \times\left[\left(K_{\mathrm{mo}}+K_{\mathrm{re}}\right) \sin (\varphi)+R_{\mathrm{mo}} \dot{\varphi} \cos (\varphi)\right]=I_{\mathrm{cg}} \ddot{\varphi} \\
& I_{\mathrm{cg}} \ddot{\varphi}=I_{z \mathrm{~g}} \varpi_{\mathrm{G}} \dot{\theta}-2 h_{\mathrm{c}}^{2}\left[\left(K_{\mathrm{mo}}+K_{\mathrm{re}}\right) \varphi+R_{\mathrm{mo}} \dot{\varphi}\right] \\
& \quad I_{\mathrm{cg}} \ddot{\varphi}=I_{z \mathrm{~g}} \varpi_{\mathrm{G}} \dot{\theta}-\left(K_{\mathrm{mo}}+K_{\mathrm{re}}\right) \varphi 2 h_{\mathrm{c}}^{2}-R_{\mathrm{mo}} \dot{\varphi} 2 h_{\mathrm{c}}^{2}
\end{aligned}
$$

$$
I_{\mathrm{cg}} \ddot{\varphi}=I_{z \mathrm{~g}} \varpi_{\mathrm{G}} \dot{\theta}-\left(K_{\mathrm{mo}}+K_{\mathrm{re}}\right) \varphi 2 h_{\mathrm{c}}^{2}-R_{\mathrm{mo}} \dot{\varphi} 2 h_{\mathrm{c}}^{2}
$$

Nous allons maintenant utiliser le formalisme de Laplace afin de réécrire ces équations linéarisées sous forme d'équations d'états

$$
\begin{aligned}
I t \theta p^{2}= & -R_{\mathrm{l}} \nu^{2} \theta p+R_{\mathrm{l}} z_{1} \nu p-K_{\mathrm{l}} \nu^{2} \theta \\
& +z_{1} K_{\mathrm{l}} \nu-I_{z \mathrm{~g}} \varpi_{\mathrm{G}} \varphi p-R t \theta p-K t \theta \\
I_{\mathrm{cg}} \varphi p^{2}= & I_{z \mathrm{~g}} \varpi_{\mathrm{G}} \theta p-\left(K_{\mathrm{mo}}+K_{\mathrm{re}}\right) \varphi 2 h_{\mathrm{c}}^{2}-R_{\mathrm{mo}} \varphi p 2 h_{\mathrm{c}}^{2}
\end{aligned}
$$

De l'équation (B.4) nous sortons l'expression de $\varphi$ que nous replaçons dans l'équation (B.3)

$$
\begin{aligned}
I_{\mathrm{cg}} \varphi p^{2} & =I_{z \mathrm{~g}} \varpi_{\mathrm{G}} \theta p-\left(K_{\mathrm{mo}}+K_{\mathrm{re}}\right) \varphi 2 h_{\mathrm{c}}^{2}-R_{\mathrm{mo}} \varphi p 2 h_{\mathrm{c}}^{2} \\
\varphi & =\frac{I_{z \mathrm{~g}} \cdot \varpi_{\mathrm{G}} \theta p}{I_{\mathrm{cg}} p^{2}+R_{\mathrm{mo}} p 2 h_{\mathrm{c}}^{2}+\left(K_{\mathrm{mo}}+K_{\mathrm{re}}\right) 2 h_{\mathrm{c}}^{2}}
\end{aligned}
$$

Nous obtenons :

$$
\begin{aligned}
I t \theta p^{2}= & -R_{\mathrm{l}} \nu^{2} \theta p+R_{\mathrm{l}} z_{1} \nu p-K_{1} \nu^{2} \theta+z_{1} K_{1} \nu \\
& -I_{z \mathrm{~g}} \varpi_{\mathrm{G}} \varphi p-R t \theta p-K t \theta \\
I t \theta p^{2}+ & R_{\mathrm{l}} \nu^{2} \theta p+K_{\mathrm{l}} \nu^{2} \theta+R t \theta p+K t \theta \\
& +\frac{\left(I_{z \mathrm{~g}} \varpi_{\mathrm{G}}\right)^{2} \theta p^{2}}{I_{\mathrm{cg}} p^{2}+R_{\mathrm{mo}} p 2 h_{\mathrm{c}}^{2}+\left(K_{\mathrm{mo}}+K_{\mathrm{re}}\right) 2 h_{\mathrm{c}}^{2}} \\
& =R_{\mathrm{l}} z_{1} \nu p+z_{1} K_{1} \nu
\end{aligned}
$$

Voir équation ci-dessus 


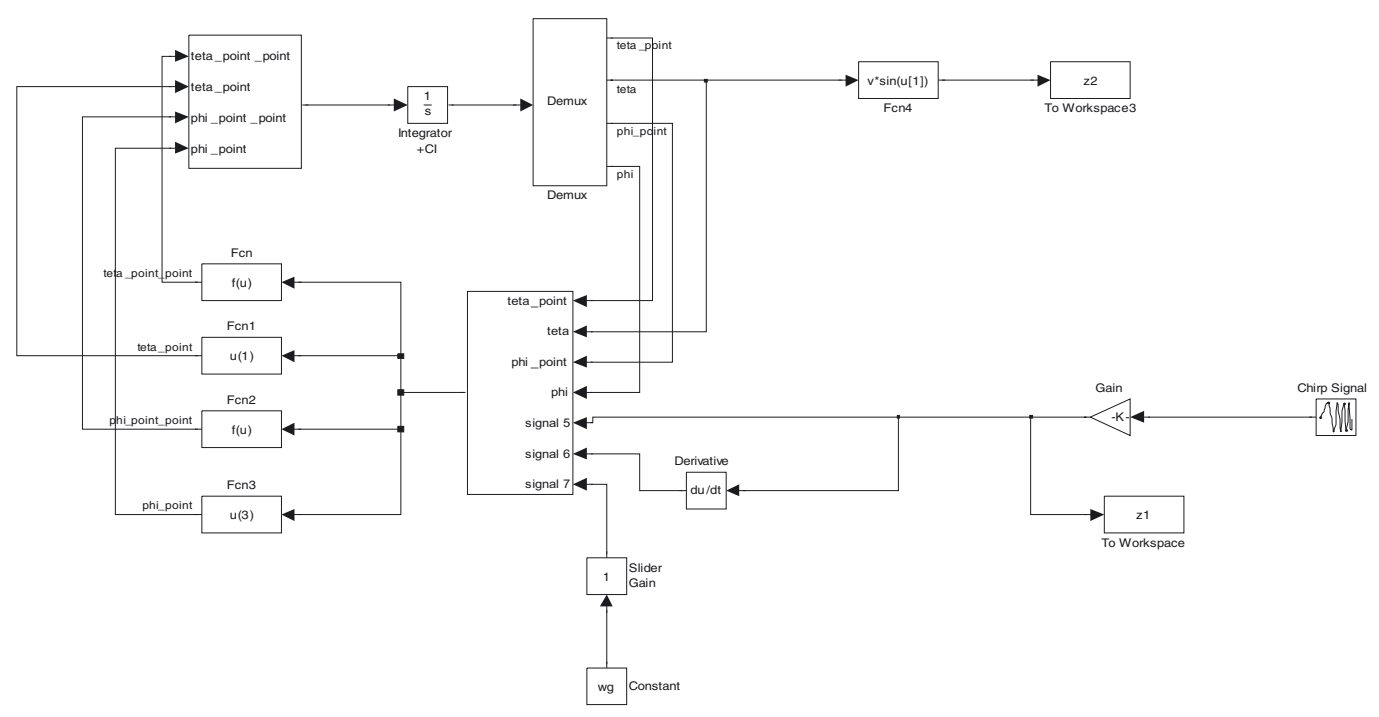

Fig. C1. Modèle d'état implémenté dans Similink.

\section{Annexe C : Représentation d'état des équations}

Pour la représentation d'état des équations, nous exprimons les dérivées secondes de $\theta$ et $\varphi$ en fonction de leurs dérivées inférieures et des autres paramètres.

De l'équation (1) :

$$
\begin{aligned}
(\nu \cos (\theta)+h \sin (\theta)) & \left(-R_{\mathrm{l}}\left(\dot{z}_{2}-\dot{z}_{1}\right)-K_{\mathrm{l}}\left(z_{2}-z_{1}\right)\right) \\
& -I_{z \mathrm{~g}} \varpi_{\mathrm{G}} \dot{\varphi} \cos (\varphi)=I t \ddot{\theta}+R t \dot{\theta}+K t \theta
\end{aligned}
$$

nous tirons :

$$
\begin{aligned}
\ddot{\theta}=\frac{1}{I t}((\nu \cos (\theta)+h \sin (\theta)) & \left(-R_{\mathrm{l}}\left(\dot{z}_{2}-\dot{z}_{1}\right)\right. \\
& \left.\left.-K_{\mathrm{l}}\left(z_{2}-z_{1}\right)\right)-R t \dot{\theta}-K t \theta\right)
\end{aligned}
$$

De l'équation (2) :

$$
\begin{aligned}
& I_{z \mathrm{~g}} \varpi_{\mathrm{G}}(\dot{\theta} \cos (\theta) \cos (\varphi)+\dot{\varphi} \sin (\theta) \sin (\varphi)) \\
& \quad-h_{\mathrm{c}}\left[2 h_{\mathrm{c}} \cos (\theta) \cos (\varphi)+l_{\mathrm{c}} \sin (\theta)\right] \\
& \quad \times\left[\left(K_{\mathrm{mo}}+K_{\mathrm{re}}\right) \sin (\varphi)+R_{\mathrm{mo}} \dot{\varphi} \cos (\varphi)\right]=I_{\mathrm{cg}} \ddot{\varphi}
\end{aligned}
$$

nous tirons :

$\ddot{\varphi}=\frac{1}{I_{\mathrm{cg}}}\left(\begin{array}{l}I_{z \mathrm{~g}} \varpi_{\mathrm{G}}(\dot{\theta} \cos (\theta) \cos (\varphi)+\dot{\varphi} \sin (\theta) \sin (\varphi)) \\ -h_{\mathrm{c}}\left[2 h_{\mathrm{c}} \cos (\theta) \cos (\varphi)+l_{\mathrm{c}} \sin (\theta)\right] \\ \times\left[\left(K_{\mathrm{mo}}+K_{\mathrm{re}}\right) \sin (\varphi)+R_{\mathrm{mo}} \dot{\varphi} \cos (\varphi)\right]\end{array}\right)$
Nous obtenons le système suivant :

$$
\begin{aligned}
\ddot{\theta}=\frac{1}{I t}((v \cos (\theta)+h & \sin (\theta))\left(-R_{\mathrm{l}}\left(\dot{z}_{2}-\dot{z}_{1}\right)\right. \\
& \left.\left.-K_{\mathrm{l}}\left(z_{2}-z_{1}\right)\right)-R t \dot{\theta}-K t \theta\right)
\end{aligned}
$$

$$
\ddot{\varphi}=\frac{1}{I_{\mathrm{cg}}}\left(\begin{array}{l}
I_{z \mathrm{~g}} \varpi_{\mathrm{G}}(\dot{\theta} \cos (\theta) \cos (\varphi)+\dot{\varphi} \sin (\theta) \sin (\varphi)) \\
-h_{\mathrm{c}}\left[2 h_{\mathrm{c}} \cos (\theta) \cos (\varphi)+l_{\mathrm{c}} \sin (\theta)\right] \\
\times\left[\left(K_{\mathrm{mo}}+K_{\mathrm{re}}\right) \sin (\varphi)+R_{\mathrm{mo}} \dot{\varphi} \cos (\varphi)\right]
\end{array}\right)
$$

De plus de manière évidente, nous avons :

$$
\left\{\begin{array}{l}
\dot{\theta}=\dot{\theta} \\
\dot{\varphi}=\dot{\varphi}
\end{array}\right.
$$

Le modèle d'état implémenté sous Simulink est représenté figure $\mathrm{C} 1$.

\section{Références}

[1] C.M. Harris, Shock and Vibration Handbook, 4th edition, in: C.M. Harris, C.E. Crede (éd.), 1995

[2] C. Townsend, A.J. Murphy, R.A. Shenoi, A new active gyrostabiliser system for ride control of marine vehicles, University of Southampton, UK, 2007

[3] T.W.B. Kibble, Frank H Berkshire, Classical Mechanics, 5th edition, Ed. Imperial College Press, 2004

[4] H. Goldstein, C.P., Jr. Poole, J.L. Safko, Classical Mechanics, in : A. Wesley Longman (éd.), 3rd edition, 2001

[5] J.-C. Radix, Le Gyroscope et ses applications, les Presses universitaires de France, 1969 